\title{
El Dr. José María Cano y Noreña (1787-1848): de la enseñanza extra-aula a Decano universitario
}

\author{
Dr. José María Cano y Noreña (1787-1848): \\ from out-of-classroom teaching to University Dean
}

DOl: https://doi.org/10.32870/dse.v0i21.636

\author{
Gabriela Guadalupe Ruiz Briseño* \\ Ortencia Viveros Ríos** \\ Laura Catalina Díaz Robles***
}

\begin{abstract}
Resumen
Reconstruir la vida profesional de un galeno cuya actividad fue muy copiosa, resulta difícil de hacer en unas cuantas cuartillas. En esta ocasión presentamos un breve bosquejo de la trayectoria laboral de un facultativo que se destacó por su interés en introducir los estudios sobre anatomía en la formación médica, por su tenacidad en formar "médicos con conocimientos quirúrgicos" al margen de la Universidad de Guadalajara, así como por el papel que desempeñó en la conservación y propagación de la vacuna: nos referimos al Doctor José María Cano y Noreña. Ser catedrático era la más alta distinción que se podía alcanzar pues equivalía a formar parte de una elite; el cargo brindaba autoridad, prestigio social y estabilidad económica. Además, le permitía formar parte del cuerpo de doctores, es decir, el órgano de gobierno que tomaba las decisiones en el ámbito universitario. Con material que encontramos disponible en archivo, en este escrito mostramos parte de su actividad cotidiana entretejida con el acontecer universitario, local y nacional de finales del siglo XVIII y primeras décadas del XIX.
\end{abstract}

Palabras clave: enseñanza - medicina - anatomía - viruela - vacuna.

\begin{abstract}
Reconstructing the professional life of a physician whose activity was very copious is difficult to do in just a few pages. This time we present a brief outline of the career of a doctor who stood out for his interest in introducing anatomy studies in the medical training, for his tenacity in training "physicians with surgical
\end{abstract}

* Maestra en Ciencias Sociales. Profesora Docente Asociada "C". Departamento de Historia, Centro Universitario de Ciencias Sociales y Humanidades, Universidad de Guadalajara. Miembro del cuerpo académico en consolidación: "Sociedad, cultura y desarrollo". Líneas de investigación: Historia de la medicina e Historia y Educación. México. gabyru7@hotmail.com

**Doctora en Educación. Profesora Docente Titular "B". Departamento de Historia, Centro Universitario de Ciencias Sociales y Humanidades, Universidad de Guadalajara. Miembro del cuerpo académico en consolidación: "Sociedad, cultura y desarrollo". Líneas de investigación: Historia de la Educación y Docencia de la Historia. México. orteviri08@hotmail.com

*** Doctora en Ciencias Sociales. Profesora Investigadora Asociada. Departamento de Estudios en Educación. Universidad de Guadalajara. Miembro del cuerpo académico en consolidación: “Sociedad, cultura y desarrollo". México. catidr@yahoo.com.mx 
knowledge" outside the University of Guadalajara, as well as for the role he played in the conservation and distribution of the vaccine. For Dr. José María Cano y Noreña, being a professor was the highest distinction that could be achieved, equivalent to being part of elite: a position that brought with it authority, social prestige, and economic stability. In addition, it allowed him to form part of the board of doctors; that is, the governing body that made decisions at the university level. With material found in archives, in this writing we show part of his daily activity, interwoven with the university life and the local and national events of the late eighteenth and early nineteenth centuries.

Keywords: teaching - medicine - anatomy - smallpox - vaccine.

\section{Introducción}

José María Cano y Noreña nació por el año de 1787 en Guadalajara (Iguiniz, 1992: 111), su niñez trascurrió en la calma de esta ciudad comercial a la que llegaban las noticias tanto de la Revolución francesa como del surgimiento de nuevas naciones americanas, otrora colonias.

Autores como Serrera y Arias (Serrera en Muriá et. al, 1982: 105; Arias en Martínez, 2000: 27-28, 30) señalan que a partir de 1786 la Intendencia de Guadalajara ${ }^{1}$ vio duplicar el número de sus habitantes en el plazo de tres décadas, ${ }^{2}$ entre ellos se contarían también los indígenas. Además, la generalización del pago en salario a este grupo poblacional le permitió la oportunidad de gastar sus ingresos en productos provenientes del mercado. Por su parte, los sectores agrícola, industrial y artesanal tuvieron un fuerte y progresivo incremento. Toda esta producción a nivel local podía tener un nuevo escaparate, el Mercado Alcalde, construido en 1800. Los dueños de comercios formales cumplían las funciones de banca o agio, lo cual les hizo estrechar vínculos con los pequeños productores (Arias en Martínez, 2000: 22). Asimismo, las mercancías llegaban con menor dificultad a la ciudad de México debido a la creación de un servicio regular de diligencias, a la construcción de puentes y mejoramiento de las carreteras existentes (Riviére en Muriá et al., 1982: 44).

A lo anterior habría que añadir un ajuste de las funciones administrativas que afectarían a la intendencia, tales como el establecimiento del puerto de San Blas como embarcadero de mercancías en 1768, lo que eliminaba a Veracruz como el único autorizado; la instalación de un consulado propio en la ciudad a partir de 1795. Por si eso fuera poco, desde finales del siglo XVII se advertía el afianzamiento de la función de la enseñanza, por lo que no resultó sorprendente

1 La Intendencia de Guadalajara, anteriormente Provincia de Nueva Galicia, fue una de las subdivisiones administrativas del virreinato de Nueva España entre 1786 y 1821. Su superficie corresponde a los actuales estados mexicanos de Aguascalientes, Jalisco, Nayarit, Colima y la parte sur de Zacatecas. Se llamó así a partir de la división en Intendencia de Guadalajara e Intendencia de Zacatecas en 1786, según la Real Ordenanza del mismo año. Esta nueva forma de organización territorial pretendía establecer mayor claridad con respecto a la forma de gobernar, para saber con mayor precisión quién tendría que recibir las órdenes y a quiénes podrían dárselas (Muriá en Muriá et. al., 1982: 363).

2 Para la primera década del siglo, José Fernando Abascal calculó la población de la Intendencia de Guadalajara en 522,317 habitantes para los años 1800-02, y el mismo Humboldt presentó las cifras de 630,500 habitantes (Serrera en Muriá et. al., 1982: 112).

Diálopos sobre Educación año 11 | número 21 | julio-diciembre 2020 | ISSN 2007-2171 
que fuera este el momento en el que se fundara una institución de estudios superiores de gran envergadura, y una imprenta que editara los volúmenes que los jóvenes estudiantes requerirían.

No obstante la pujanza de algunos sectores económicos y sociales, las epidemias y secuelas de hambre que siguieron a la sequía de 1784 hicieron necesaria la fundación del Hospicio Cabañas o Casa de la Misericordia, así como la construcción de un nuevo edificio para el Hospital de Belén. El avance del conocimiento médico resultaba una necesidad apremiante frente a los contagios masivos, sobre todo considerando que en Francia la medicina científica iniciaba su institucionalización (Arias en Réding, 2000: 3; Ramírez, 2004: 9-11).

\section{A manera de preámbulo}

Para que Guadalajara contara con su propia universidad fue necesario transitar por 96 años de protocolos y trámites con la Corona española. Estas gestiones fueron efectuadas por diferentes instancias regionales: "primero el obispo, luego un literato amante de historia, después el Ayuntamiento, y finalmente la Real Audiencia"33 (Cárdenas, 1999: 49). Por fin, el 18 de noviembre de 1791, Carlos IV firmó el decreto de creación de la Real Universidad de Guadalajara, mismo que se recibió en esta ciudad el 26 de marzo de 1792. En la Cédula se ordenaba la incorporación de algunas cátedras que se impartían a la Universidad en el Seminario de San José, habilitando los cursos para grados mayores y la creación de cátedras nuevas como Cánones, Leyes, Medicina y Cirugía. Se trataría de una institución ceñida orgánicamente al clero secular local cuya misión sería proveer cuadros a la iglesia y viceversa (Martínez, 2007: 30).

Además, el documento disponía que, tanto al intendente como al obispo, y al maestrescuela de la catedral en quien debía recaer el cargo de Cancelario, ${ }^{4}$ les correspondería la elección del rector, para que abrieran concurso a oposición de cátedras, nombrando a jueces para elegir a los más oportunos. Una vez erigida la Universidad, su claustro redactaría sus propias Constituciones siguiendo el modelo de las de Salamanca ${ }^{5}$ y las enviara a España para su aprobación. La inauguración tuvo lugar el 3 de noviembre de 1792.

El borrador de Constituciones que elaboró el claustro tapatío fue enviado a España en 1800. La facultad médica inició actividades con las cátedras de medicina y cirugía, apegadas

3 La Real Audiencia de Guadalajara fue el más alto tribunal de la Corona española en el territorio de Nueva Galicia, Virreinato de Nueva España, y estaba subordinado inicialmente a la Real Audiencia de México. Fue establecida el 13 de febrero de 1548 en Compostela y trasladada a Guadalajara en 1560. Su función era administrar la justicia entre sus habitantes, velar por el cumplimiento de las instrucciones y ordenanzas dadas por el rey. En 1572, por lo menos en el papel, se decretó su independencia de la Audiencia de México (Parry en Muriá et. al., 1982: 267).

4 Cancelario a maestrescuela, quien era un miembro del cabildo eclesiástico y podía "enseñar por sí o por otro". Su obligación era recibir, ante el secretario de la Universidad, las inscripciones de los que pretendían obtener grados mayores de licenciado y doctor y hacer que se practicaran las diligencias establecidas por los reglamentos para ganarlos. También le correspondía lo relativo a la incorporación de los graduados por otras universidades (Peregrina,1993: 23-24).

5 Real Cédula de creación de esta Universidad expedida por el rey de España Carlos IV. España, noviembre 18 de 1791. Archivo Histórico de la Universidad de Guadalajara (AHUG). Fondo Real y Literaria Universidad de Guadalajara. Exp. 56, 5 fs. 
al plan de estudios de medicina de la Universidad de Salamanca. Sin embargo, dicho plan se ajustó a las necesidades propias, por ejemplo, se omitió la enseñanza de la cirugía que en la constitución salmantina sí estaba incluida. En Guadalajara, el órgano de gobierno interno consideró a esta cátedra como Vísperas de Medicina, para que tanto el catedrático a cargo de esta como el de cirugía, impartieran la enseñanza sustentada en las obras de Herman Boerhaave y sus discípulos, pero a falta de un tercer catedrático que impartiera Anatomía Práctica —necesaria para conocer la geografía humana en el trabajo de la clínica médica y para la enseñanza de la cirugía-, se sugería la atendiera también el encargado de vísperas. Así quedó escrito en las Constituciones, pero en la realidad el catedrático de cirugía no se asumió como el de vísperas y enseñó la cirugía —no práctica y sin antecedentes de anatomía - apoyándose en el texto de Juan de Gorter, obra que se llevaba en Salamanca. Por esta razón, a la cátedra de cirugía también se le conocía como de vísperas, y esto nos explica por qué durante este periodo de estudio es recurrente la necesidad de estudiar la anatomía práctica.

El plan de estudios de Salamanca (1771) contempla la formación de médicos y de cirujanos latinos, a Guadalajara le interesaba sólo formar a médicos, por lo tanto elaboró su plan con aquello que correspondía sólo a la formación médica, - la de cirugía estaba contemplada en la formación de los cirujanos latinos - pero aquí la de cirugía fue creada por cédula, por tanto se mantuvo, pero a falta de recursos humanos y económicos, no se cubrió lo establecido en el plan de estudios, quedando dos cátedras que estuvieron desconectadas entre sí (Horta, 1982: 83).

La Real Universidad de Guadalajara, durante su primer periodo de vida (1792-1826) solo otorgó diez grados mayores en medicina (licenciado y doctor), de los cuales cuatro se formaron como bachilleres en la Real y Pontificia Universidad de México y en la de Guadalajara recibieron los grados mayores, estos fueron los catedráticos fundadores. Los otros seis recibieron la formación completa dentro de esta Universidad.

\section{Los estudios y su pasantía}

José María Cano y Noreña fue hijo legítimo de don Lorenzo Cano y de doña María Josefa Noreña. Sus primeros estudios los realizó en el Seminario de San Juan Bautista, en Guadalajara. Ingresó a la universidad para estudiar medicina, fue uno de los 104 alumnos de medicina que hubo entre 1792 a 1821 (Peregrina, 1993: 26). Tuvo el privilegio de formarse con el Dr. Pedro Tamez, ${ }^{6}$ quien entonces fungía como catedrático de cirugía. Profesor con quien, además de aprender cono-

6 El Dr. Pedro Tamez se formó como médico en la Real y Pontificia Universidad de México. En 1798 obtuvo los grados mayores en la Real Universidad de Guadalajara y se incorporó como catedrático. Sustituyó la cátedra de cirugía, por enfermedad de su titular, desde 1807 hasta 1812. Falleció en 1814. En los distintos documentos localizados, en algunas ocasiones encontramos escrito Tamez y en otras Tamés. Nosotras usaremos la primera forma. 
cimientos quirúrgicos, aprendería lecciones de altruismo hacia la humanidad doliente, sobre todo los de más bajos recursos y en periodos de epidemias. Ortencia Viveros (1991) señala que en 1813 Tamez ya practicaba la disección de cadáveres, cosa que también debió enseñar a su pupilo en algún momento de su trayectoria académica o profesional. Otro de sus profesores fue el Dr. Mariano García de la Torre, ${ }^{7}$ catedrático de Prima de Medicina.

En cuanto a los grados, la Universidad otorgó tres, el de bachiller, licenciado y doctor. Para obtener el de bachiller en medicina era indispensable contar con el grado de bachiller en artes y haber aprobado cuatro cursos, cada uno con dos cátedras, Prima y Vísperas de Medicina, y haber sustentado un acto mayor y uno menor (Cárdenas, 1999: 95). Dicho grado era el equivalente a ser considerado "pasante" en dicha formación; la mayoría de los egresados ejerció con este. El Dr. Cano y Noreña obtuvo el grado de bachiller el 30 de junio de $1810 .^{8}$

Para obtener el grado de licenciado era necesario el de bachiller en medicina; para adquirirlo no se exigían nuevos cursos pero sí cumplir con la pasantía, es decir, tres años de práctica junto a un profesor de reconocida experiencia, luego ser examinado y aprobado por el Tribunal del Protomedicato para ejercer la medicina y sustentar un acto mayor: la "repetición" ${ }^{\prime \prime}$ en el aula general de la Universidad. La obtención del grado de doctor se limitaba a que el sustentante debía tener un padrino cuyo papel era acompañarlo.

Más bien su significado debe hallarse en el afán de una élite académica que busca diferenciarse del bachiller común, tanto por prestigio como para tener mayores posibilidades de conseguir un alto XVI puesto en la iglesia o en la burocracia real. De hecho, ya desde el siglo, los doctores se adueñaron de la corporación universitaria (gobierno, cátedras y finanzas), buscando desde tal espacio una pronta promoción a cargos encumbrados y altas rentas (Aguirre, 2003: 80-81).

Aparte del proceso administrativo, la obtención del grado implicaba, entre otras cosas, una erogación monetaria que era muy alta; y finalmente, la búsqueda de encumbrados padrinos y mecenas que en la mayoría de los casos se hallaba entre las máximas autoridades del virreinato, en los prebendados de catedral, o bien, en familias nobles o ricos comerciantes. "Los doctores significaron el sector más elitista dentro del universo de graduados y, evidentemente, el más reducido también. Por supuesto que esta élite académica, en su afán de salvaguardar su jerarquía, cuidaba lo mejor posible los mecanismos necesarios para el otorgamiento de los grados de

7 El Dr. Mariano García de la Torre fue el primer catedrático de Prima de Medicina en la Real Universidad, desde 1762 fungió como médico titular del Hospital Real de San Miguel de Belén, además fue el único médico que tuvo el carácter propio de médico municipal durante la dominación española, y permaneció en el cargo más de medio siglo (Gaceta Municipal de Guadalajara, 1917: 163-166).

8 Libro primero de asientos de grados de bachiller en Medicina durante el periodo del 9 de julio de 1796 al 13 de agosto de 1859. AHUG. FRyLUG. Caja 39, f. 6 vta.

9 La repetición era un acto mayor que consistía en una disertación de una hora, cuyo tema se escogía libremente entre todas las materias de la facultad..." (Cárdenas, 1999: 97). 
licenciado y de doctor" (Aguirre, 2003: 81). Quizá esto explique porqué, en el periodo estudiado, en la Universidad solo se otorgaron diez grados mayores.

Se enseñaba bajo preceptos escolásticos, que era la filosofía de la época medieval. Parafraseando a Martínez (2007: 27), señalaremos que dicho pensamiento tuvo sus fuentes reproductivas durante la Colonia en dos instancias: la iglesia (y con ella los seminarios conciliares) y la Universidad. No había mucha diferencia entre ambas, añade el autor. La medicina, que en Europa reflejaba el carácter avanzado de las ciencias ilustradas, en la Universidad se ejerció con un formato pedagógico anacrónico, dogmático y su ejercicio era decadente.

Los galenos antecesores a Cano y Noreña, que obtuvieron grados mayores en medicina y decidieron continuar su profesión en la docencia, como Ignacio Otero y Patiño, ${ }^{10}$ José María Jaramillo ${ }^{11}$ y José María Ylisaliturrii, ${ }^{12}$ se trasladaron a México a cubrir su pasantía, realizaron estudios de anatomía práctica en el Hospital de San Andrés y tomaron un curso de botánica en el Jardín Botánico de aquella ciudad. Una vez que cubrieron su pasantía en México y el Protomedicato les dio el reconocimiento, regresaron a la ciudad. Otero y Jaramillo fueron compañeros en la facultad médica, egresaron juntos como bachilleres el 19 y 20 de junio de 1798 respectivamente. Ylisaliturri egresó en septiembre de $1800 .{ }^{13}$ Con excepción de Jaramillo, que obtuvo sus grados mayores en 1805 y se fue a Zacatecas a ejercer su profesión, los otros dos continuaron su trayectoria en la Universidad haciendo sustituciones a las cátedras cuando los catedráticos enfermaban o solicitaban licencias, recibieron sus grados mayores en 1809 y 1813 , cuando se abrieron concursos para una cátedra.

José María Cano y Noreña, al igual que el resto de los egresados que no podían sufragar los costos de trasladarse a la ciudad de México para cubrir su pasantía, se quedaron en la ciudad para ejercerla en el Hospital de Belén o el de San Juan de Dios, y quizás unos cuantos más que no pretendían hacer carrera por una cátedra, decidieron ejercer la medicina solo con el grado de bachiller.

Cano y Noreña se incorporó al Hospital de Belén al lado de García de la Torre, desempeñando el cargo de practicante de medicina en las salas de hombres y mujeres. Además — señala el mismo Cano-, persuadiéndose de que era necesario agregar a los conocimientos médicos los Chirurgicos, ${ }^{14}$ con el cirujano mayor de dicho nosocomio de Belén procedió a practicar conocimientos que le fueron muy útiles cuando en 1810 estalló la guerra de independencia.

10 Ejercicios literarios del doctor Ignacio Otero, presentados en el concurso por la cátedra de prima de medicina, s/f. AHUG. FRyLUG. Caja 8, Exp. 164.

11 Ejercicios literarios del doctor José María Jaramillo presentados en el concurso de la cátedra de vísperas de medicina, Guadalajara, s/f. AHUG. FRyLUG. Caja 8, Exp. 172.

12 Ejercicios literarios del doctor José María llisaliturri, presentados en el concurso por la cátedra de prima de medicina, Guadalajara, s/f. AAHUG. FRyLUG. Caja 9, Exp. 173, fs. 17-18.

13 Libro primero de asientos de grados de bachiller en medicina. Libro: 31, Guadalajara: 1796-1859, AHUG. FRyLUG. Caja 39, fs. 200. 14 Es decir, de cirugía.

Diálo pos 
Un informe del año de 1814, sobre el estado en que se encontraba el Hospital de Belén, hacía énfasis en el excesivo número de pacientes y la poca atención que podía dar el médico titular y nos proporciona el número de practicantes registrados en dicho nosocomio:

Lista de los individuos empleados en el hospital general de San Miguel de Belén...

Medico titular: Dr. Don Mariano Torre

Practicante mayor de medicina: Bachiller Don José María Portugal

Dos practicantes segundos de medicina: Don Hernando Cabrera y Don Ramón Salcedo.

Cirujano Mayor: Don José María Herrera

Practicante menor de cirugía: Don José María Cano

Practicante segundo de Cirugía: Don Ygnacio Gutiérrez [... $]^{15}$

De hecho, después de un año de práctica, el excesivo trabajo no había disminuido, más bien al contrario. Ante las milicias que empezaban a formarse, el Cirujano Mayor solicitó al Superior Gobierno, encabezado por el general José de la Cruz, contar con un ayudante, por lo que Cano desempeñó ambas funciones, como pasante médico y practicante quirúrgico "sin desatender el de asistir al lado de su maestro el Dr. Torre, tanto que logró la satisfacción muchas veces de desempeñarle su visita en sus ausencias y enfermedades hasta que murió [en 1815]".16 En 1811, un brote de fiebre amarilla o vómito prieto se extendió desde Puebla a todo el virreinato, ${ }^{17}$ epidemia que asoló a la ciudad entre los años de 1813 y 1814, entonces el gobierno del estado habilitó al Dr. Cano para auxiliar en tal evento (Iguiniz, 1992: 11-12).

Su intervención como médico la entendemos en el sentido de su deber de acuerdo con el juramento hipocrático, y porque la Universidad, como institución formada por la Corona, durante la independencia estuvo en contra de Hidalgo, se dio una campaña de desprestigio al movimiento y exhortos a defender el Reino, participación política e incluso militar, con panfletos y apoyos económicos (Martínez, 2007: 32-33).

\section{La pretensión por la docencia universitaria}

Durante el tiempo que Cano realizó su pasantía, los primeros catedráticos universitarios comenzaron a dejar sus cátedras por jubilación, y posteriormente por fallecimiento. Esto motivó que varios bachilleres entraran al concurso por alguna de las cátedras. Cano no concursó en

15 Informe sobre la relación que guarda el hospital de Belén de acuerdo con su plan de Gobierno sobre las rentas y fincas que posee, el orden de las cuentas, provisión y consumo de víveres, despacho de medicamentos, número de empleados, sueldos, estado físico del edificio, la asistencia espiritual y corporal a los enfermos. Guadalajara, 13 de marzo de 1814. Archivo Histórico del Arzobispado de Guadalajara (AHUG). Caja 4, progresivo 61, Expedientes 3, fs. 26.

16 Sobre la provisión de la cátedra de víspera de medicina en el Dr. Don José María Cano, por muerte del Dr. Don José María Jaramillo. Guadalajara, 1822. AHUG. FRyLUG Exp. 174, f. 23.

17 Afirmación extraña, según Viesca (2010: 50), pues la altitud de Puebla no permite la sobrevivencia del aedes aegypti, y es la misma situación para gran parte del territorio mexicano. 
las primeras convocatorias pues aún no terminaba su periodo de pasantía, además, quienes se acomodaron en estos cargos fueron los bachilleres egresados de las primeras generaciones, quienes contaban con más tiempo de experiencia y méritos.

Para noviembre de 1812, Mariano García de la Torre, catedrático de Prima, solicitó su jubilación después de veinte años de servicio. Pedro Tamez, que estaba sustituyendo la cátedra de cirugía por enfermedad de Ignacio Brizuela, su titular, la dejó para concursar por la sustitución a la Prima de Medicina. El 15 de noviembre la Universidad convocó a concurso para cubrir lo que restaba del cuadrienio de la de cirugía - que Tamez había dejado vacante - y fue otorgada al ya doctor Ignacio Otero. El 17 de noviembre del mismo año se abrió concurso por la sustitución de la de Prima, ocupando ese lugar Pedro Tamez.

Tres meses después, el 15 de febrero de 1813 falleció Ignacio Brizuela, por lo que el día 26 del mismo mes y año la plaza de Cirugía se puso a concurso, pero ahora en propiedad. Tamez dejó la sustitución de la de Prima y concursó por la propiedad la de Cirugía, que le fue otorgada. Entonces se puso a concurso, el 22 de diciembre, la sustitución la de Prima que había dejado Tamez, y la ganó Ignacio Otero.

El 7 de marzo de 1814 falleció Pedro Tamez. El 10 de marzo se puso a concurso en propiedad la cátedra de cirugía. Ignacio Otero dejó la sustitución de la de Prima para obtener en propiedad la que dejaba su colega. A su vez, el 14 de julio de ese año José María Ylisaliturri concursó y ganó la sustitución de la cátedra de Prima, que había dejado Otero. Ylisaliturri acababa de obtener los grados mayores en enero de 1813, precisamente para tener más posibilidades de lograr una cátedra.

El 18 de mayo de 1815 falleció Mariano García de la Torre, quien fuera el titular de la cátedra de Prima, entonces jubilado. El 20 de mayo se puso a concurso en propiedad la cátedra de Prima. Otero dejó la de Cirugía y ganó en propiedad la de Prima. El 25 de octubre del mismo año se concursó en propiedad la de Cirugía que acababa de dejar Otero. La plaza fue otorgada a José Ma. Ylisaliturri.

Tras el fallecimiento del Dr. Mariano García de la Torre, Noreña participó como opositor en estos dos últimos concursos. Aunque sabía que sus posibilidades de ganar eran mínimas frente a la antigüedad y grados de los oponentes. Antes de que fuera el concurso por la de cirugía, Cano solicitó los grados mayores, pero fue apelado por el bachiller José María Gómez de Portugal, por derecho de antigüedad, para obtener los primeros grados pues él había egresado en el año de 1808.

Gómez de Portugal contó con el apoyo del claustro universitario y compañeros médicos, sus padrinos de grado fueron el Dr. Toribio González, Prebendado de la catedral, y los doctores Ignacio Otero y José María Ylisaliturri. ${ }^{18}$ Mientras que a Cano, en su examen por el de licenciado

18 Certificación hecha por el secretario sobre los grados mayores en medicina que pretende recibir José María Portugal en esta Universidad. Guadalajara, 14 octubre de 1815. AHUG. FRyLUG. Exp. 104, fs. 12.

Diálo Pos sobre Educación año 11 | número 21 | julio-diciembre 2020 | ISSN 2007-2171 
nadie lo apadrinó, por el de doctor fue el general y gobernador José de la Cruz. Aun así, durante el concurso por la cátedra de cirugía fue en calidad de bachiller, pues Portugal obtuvo los grados el 23 de noviembre y 3 de diciembre, y Cano los había obtenido el 30 de noviembre y 24 de diciembre respectivamente.

Aunque no obtuvo ninguna clase en estos concursos, el haber obtenido los grados mayores lo incorporaba como catedrático de la Universidad y lo hacía miembro del claustro de doctores. Para ello dejó el cargo de cirujano de los departamentos de cirugía de tropa, paisanos y mujeres, ocupando la plaza de primer médico del Hospital de Belén que había dejado García de la Torre, otorgada por el mismo gobernante José de la Cruz. ${ }^{19} \mathrm{El}$ reconocimiento por parte del Protomedicato de México lo obtuvo el 24 de marzo de 1817 (Horta, 2002: 86).

El Dr. Cano mostró un gran interés por el desarrollo de la medicina, y en especial por la anatomía. En 1817, cuando llegaron las Constituciones propias, ratificadas y avaladas por la Corona española en el plan de estudios para medicina, se estipulaba que si los fondos lo permitían se estableciera una cátedra de anatomía. Noreña, como miembro del claustro de doctores, el 29 de agosto de 1818 solicitó al claustro universitario se le concediera la lectura de la cátedra de anatomía, sin más renta que la que el rector autorizara, pero la respuesta fue negativa, primero porque dicha plaza se tenía que proveer mediante concurso, y segundo, por la escasez de fondos. ${ }^{20}$ Ante esta respuesta, Cano Noreña nos refiere en sus ejercicios literarios que él propuso:

[...] al señor Rector y claustro su erección prestándose a servirla sin estipendio alguno, y costeando de su bolsa los gastos que trae consigo, como herramientas, láminas, cirujano que hiciera las operaciones. Con sólo la condición que se le abonara el tiempo para su jubilación con arreglo a estatuto; cuya propuesta con dolor suyo no fue admitida a pesar de los muchos votos que tuvo a su favor, por creer el mayor número que cuando vacase dicha cátedra no habría sujeto que tuviese igual generosidad [...]. ${ }^{21}$

Con ese argumento, en la Universidad nunca se impartió este conocimiento. Como bien señala Cárdenas (en Chavoya, 2007: 45), las cátedras se basaban en autoridades centenarias, las clases eran en latín, la Inquisición y la Corona española en conjunto impedían la llegada de libros modernos; a pesar del movimiento insurgente que se desarrollaba en ese momento, la institución en cuestión mostraba señales de estancamiento académico.

Para 1819, Ignacio Otero pidió dos licencias para ausentarse de sus clases de la Universidad por unos meses. Cano lo sustituyó en una ocasión; para 1820 José María Jaramillo regresó de

19 Sobre la provisión de la cátedra de víspera de medicina en el Dr. Don José María Cano, por muerte del Dr. Don José María Jaramillo. Guadalajara, 1822. AHUG. FRyLUG. Exp. 174, f. 23 y 23 vta.

20 El doctor don José María Cano y Noreña sobre que se le permita leer la cátedra de anatomía en los términos que propone. Guadalajara, 1818, AHUG. FRyLUG. Libro 11 del claustro de doctores, fs. 63-63 v.

21 Sobre la provisión de la cátedra de víspera de medicina en el Dr. Don José María Cano, por muerte del Dr. Don José María Jaramillo. Guadalajara, 1822. AHUG. FRyLUG. Exp. 174, f. 24. 
Zacatecas a la ciudad y se incorporó como catedrático al claustro universitario y lo sustituyó el resto del tiempo. El 23 de octubre de 1821, Ignacio Otero renunció a la cátedra de Prima debido a problemas de tos que le impedían leer en clase. Para el 27 de noviembre de 1821 se puso a concurso en propiedad la cátedra de Prima; el movimiento estaba claro, tras el concurso fue otorgada a Ylisaliturri el 22 de diciembre de ese año. Después se concursó en propiedad la de cirugía, y quien la obtuvo fue José María Jaramillo. Cano no participó en estos concursos, sabía que si opositaba no tenía oportunidad de éxito.

Meses más tarde, en mayo de 1822 falleció el Dr. José María Jaramillo y, al declararse vacante la cátedra de vísperas de medicina, Cano y Noreña la concursó y se le otorgó en propiedad el 11 de julio de 1822. ${ }^{22}$ En su relación de méritos para el concurso de esta cátedra dejaba claro su interés en la formación de estudiantes, aludiendo a una academia extra-aula que él mismo regenteaba con estudiantes de medicina:

Estando persuadido, cuan necesaria era la emulación para el aprovechamiento de la juventud, estableció en su casa hace siete años y permanece aun al presente una academia nocturna la cual ha fomentado de distintos modos, ya prestando sus débiles conocimientos en aquellos casos en que la juventud inexperta no puede penetrar, ya poniendo a su disposición una biblioteca razonable que ha adquirido procurando las obras más modernas y selectas médicas; encargándolas a México y a Madrid, ya también dando lecciones de Anatomía por medio de láminas; cuya colección tiene a disposición de los interesados y ya en fin quitando con el trato franco y familiar, el temor servil con que los jóvenes ponen trabas a su ilustración... Tiene el honor de tener algunos discípulos entre los cuales, tres están ya examinados. ${ }^{23}$

En abril de 1821, antes de que Ylisaliturri dejara la cátedra de cirugía, tuvo un altercado con un estudiante de nombre José Antonio López. Ylisaliturri pedía la expulsión del alumno por alterar el orden entre sus compañeros. El argumento que el estudiante dio al rector fue que el catedrático los obligaba a estar presentes en el aula quince minutos antes de la hora señalada y que los hacía quedarse en el salón hasta media hora después del término:

[...] nosotros que vimos la ninguna obligación de ceder a esta especie de capricho, no lo hemos hecho, de aquí ha venido el despropasarse nuestro catedrático, tratándonos de mal casados y otras expresiones nada decorosas. En las disputas escolásticas si proponemos alguna dificultad, contra sus opiniones, sufrimos el mismo ultraje [...]. ${ }^{24}$

22 Sobre la provisión de la cátedra de víspera de medicina en el Dr. Don José María Cano, por muerte del Dr. Don José María Jaramillo. Guadalajara, 1822. AHUG. FRyLUG. Exp. 174, f. 24. 23 lbíd.

24 Solicitud del rector a los catedráticos de la Nacional Universidad de Guadalajara sobre informar de la conducta del bachiller José Antonio López. Guadalajara, 1821. AHUG. FRyLUG. Caja 7, Exp. 141, fs. 14.

Diólo pos 
Otero y Jaramillo respondieron que el alumno se ausentaba de sus clases pues también estaba estudiando teología, pero que era buen estudiante y nunca causó problemas. Cano respondió que el tiempo que estuvo sustituyendo la cátedra le encontró un regular talento, "[...] que en las conferencias que de noche mantengo en mi casa, es uno de los más puntuales, y que se explica mejor. Que en orden a su conducta para con los concursantes y catedráticos, observé que es demasiado bilioso, un poco enérgico, en términos de pasar altanero".25

Los alumnos tenían contacto con el Dr. Cano mientras se formaban como médicos, como pupilos de su academia nocturna, como alumnos cuando empezó a impartir la cátedra de vísperas y, como pasantes cuando asistían al Hospital de San Miguel a realizar sus prácticas de pasantía.

En este periodo también desempeñó funciones administrativas dentro de la misma Universidad, fue nombrado consiliario durante los bienios de $1819-1821^{26}$ y 1825-1827, ${ }^{27}$ aunque este último no lo concluyó debido al cierre de la Universidad pues cuando Iturbide abdicó, luego de un efímero periodo imperial, dicho centro de estudios mantuvo sus actividades académicas y su jerarquía "sin permitir que penetraran en su régimen interno los factores liberales, que ya se desplegaban en el ámbito político. Incluso se negaron sus miembros a jurar la constitución promulgada en noviembre de 1824, hasta que le fueron suprimidos los preceptos que autorizaban al gobierno a supervisar los gastos del culto".

A la par, el Dr. José María Cano se desempeñó en diferentes cargos públicos en el Ayuntamiento de la ciudad de Guadalajara, así como para el Superior Gobierno, entre ellos podemos destacar los de: primer médico de la Junta de Sanidad, ${ }^{28}$ cargo que ocupó en diferentes ocasiones; como regidor y juez de hospitales y escuelas, del 1 de enero al 24 de diciembre de 1821 (López, 1988: 245-246); fue miembro de la Junta Superior de Sanidad en $1824^{29}$ y Presidente de la Junta de Sanidad, el 4 de enero de $1827 .{ }^{30}$ Fue nombrado por el Superior Gobierno para el reconocimiento de los militares que no estuvieran capacitados para formar parte del ejército; este empleo lo sirvió desde 1815 hasta 1822, también sirvió la plaza de médico del Imperial Cuerpo de Artillería. ${ }^{31}$

Noreña, fiel a sus creencias, fue un arduo defensor de la monarquía española como miembro de la Junta Patriótica (Iguíniz, 1992: 111-112) y regidor del Ayuntamiento. El 20 de marzo

25 lbíd.

26 Libro primero de claustros, Guadalajara, 1819. AHUG. FRyLUG. Fs. 68 vta.

27 Libro segundo de claustro de consiliarios, Guadalajara, 1825. AHUG. FRyLUG. Caja 29, fs. 22 y 22 vta.

28 Sobre la provisión de la cátedra de víspera de medicina, en el Dr. Don José María Cano, por muerte del Dr. Don José María Jaramillo. Guadalajara, 1822. AHUG. FRyLUG. Exp. 174, f. 23 vta.

29 Documento sin título. Archivo General de Guadalajara (AGG), paquete 42, leg. 242.

30 Actas de cabildo. Guadalajara 1827. AGG. A 4/827, Leg. 1.

31 Sobre la provisión de la cátedra de víspera de medicina, en el Dr. Don José María Cano, por muerte del Dr. Don José María Jaramillo. Guadalajara, 1822, AHUG. FRyLUG. Exp. 174, f. 24. 
de 1821, junto con los demás miembros del Ayuntamiento - entre ellos el Dr. Victoriano Mateos-, hizo una proclama exhortando a los habitantes de Guadalajara a guardar fidelidad al rey y a la monarquía española (Villaseñor, 1983: 162). Fue comisionado para colectar el donativo voluntario para el socorro del Ejército Imperial, y se le comisionó para la recolección del empréstito que hizo la ciudad para el embarque de tropas expedicionarias, en cuyos préstamos y donativos fue de los primero que aportaron, no obstante - según Cano- estar cargado de una numerosa familia. ${ }^{32}$

En 1825, Prisciliano Sánchez asumió la gubernatura e inició su régimen dentro de una organización político-administrativa federalista, además pugnó por que el Estado se abrogase el derecho de impartir la educación, de acuerdo con las prevenciones constitucionales. La resistencia que opuso el claustro a las medidas oficiales llevó a que el gobierno cerrara la Universidad el 18 de enero de 1826, después de que el hecho fue discutido y aprobado por la Legislatura local (Peregrina, 1993: 28).

\section{Clausura de la Universidad y creación del Instituto de Ciencias del Estado} Para Martínez (2007: 27), la Universidad de Guadalajara no llegó para contribuir a que los criollos y peninsulares obtuvieran una calificación profesional que los insertara en el desarrollo productivo, sino que "se orientaba a proveer de cuadros a los viejos estamentos privilegiados, que eran secuela de una política concebida para transplantar la grandeza del reino español [léase régimen feudalizante] a América." Los perfiles a producir debían ser teólogos y legistas religiosos, después abogados, y por último médicos.

Para este autor, la Universidad y su erudición dogmática no concordaban con una sociedad en ebullición, en incesante transición y solo representaban a un sector de la sociedad neogallega, cercano en sus usos y costumbres al viejo régimen.

Al consumarse la Independencia en 1821 el claustro prestó juramento al Plan de Iguala casi de inmediato [...] dejó de llamarse Real otorgándose el título de Nacional Universidad de Guadalajara, pero sus constituciones eran de institución regia, por ejemplo la norma que establecía que quienes ingresaran no debían ser traidores a la Corona, se conservó durante un tiempo hasta que se cambió por el de "no haya sido traidor a la patria, ni a las autoridades debidamente establecidas". Al proclamarse en $1822 \mathrm{el}$ Primer Imperio, el Claustro mandó acuñar una medalla conmemorativa, aceptó al gobierno de Iturbide, pues. Con el establecimiento de la Primera República Federal en 1824, no se hizo ningún pronunciamiento público ni se discutió en el claustro [...] La Universidad no aceptó jurar la constitución del Estado de Jalisco, porque el artículo séptimo ponía a la Iglesia local bajo el control administrativo del gobierno, todavía se sentía un ala de la Iglesia (Martínez, 2007: 34).

32 Sobre la provisión de la cátedra de víspera de medicina, en el Dr. Don José María Cano, por muerte del Dr. Don José María Jaramillo. Guadalajara, 1822. AHUG. FRyLUG. Exp. 174, f. 24. 
El hecho de introducir la cirugía como materia de enseñanza, entre otras singularidades, no es suficiente para concebirla como una institución ilustrada ni vinculada a los sectores que avanzaban hacia el desarrollo productivo de la región (Martínez, 2007: 35). En parte, estas son algunas de las razones que nos explican que en enero de 1826 Prisciliano Sánchez presentara al Congreso un proyecto de ley sobre instrucción pública, mismo que fue aprobado el 29 de marzo del mismo año, pero antes fue decretada la clausura de la Universidad de Guadalajara junto con el Colegio de San Juan.

Con el cierre de esta casa de estudios se abrió el Instituto de Ciencias, lo que marcó una ruptura con la enseñanza tradicional que la Universidad impartía. El Plan de Instrucción Pública presentaba cuatro grados de enseñanza; el cuarto nivel, que es el que nos ocupa, se refiere al Instituto de Ciencias del Estado (Cárdenas, 1999: 187, 193).

El Instituto fue inaugurado el 14 de febrero de 1827. Con la expedición de este nuevo plan se inició una creciente presencia de extranjeros en los espacios educativos. Así, por ejemplo, tenemos que para las secciones médicas fue contratado el francés Guillermo Faget. También hubo profesores y facultativos que llegaron de México como el farmacéutico Manuel Ocampo, responsable de la cuarta sección (De la Torre, 2006: 99-199), y muchos otros practicantes de la medicina como José Luis Verdía, Francisco Torres, Francisco Garibay, ${ }^{33}$ Jesús Rojas. $^{34}$

Impartieron docencia en el Instituto de Ciencias

\begin{tabular}{|l|c|l|}
\hline \multicolumn{1}{|c|}{ Sección: } & Asignatura: & \multicolumn{1}{c|}{ Profesores: } \\
\hline CUARTA: & Química & Manuel Ocampo \\
\hline NOVENA: & $\begin{array}{c}\text { Anatomía General Descriptiva. } \\
\text { Patología. } \\
\text { Cirugía Teórica }\end{array}$ & $\begin{array}{l}\text { Guillermo Faget } \\
\text { Profesor Propietario de la 9a Sección } \\
\text { José Luis Verdía, Manuel Ocampo } \\
\text { José María Cano y Noreña }\end{array}$ \\
\hline DÉCIMA: & $\begin{array}{c}\text { Fisiología } \\
\text { Higiene } \\
\text { Medicina Legal }\end{array}$ & $\begin{array}{l}\text { Francisco Torres Prof. Honorario de la } \\
\text { 10a Sección } \\
\text { José María Cano y Noreña }\end{array}$ \\
\hline \multicolumn{2}{|c|}{\begin{tabular}{c} 
Práctica de Medicina \\
\hline
\end{tabular}} & Francisco Garibay \\
\hline TERCERA: & Retórica, Lógica y Física & José María Ylisaliturri \\
\hline OCTAVA: & $\begin{array}{c}\text { Derecho Canónico, Historia } \\
\text { Eclesiástica y Concilios }\end{array}$ & José Luis Verdía
\end{tabular}

33 Egresó de la Universidad de Guadalajara el 19 de julio de 1823. En Libro primero de asientos de grados de bachiller en medicina. Guadalajara, 1796-1859. AHUG. FRyLUG. Caja 39, libro 31, fs. 202.

34 En el periodo comprendido de 1792 a 1835, a partir de diversas fuentes elaboramos un Padrón de 43 personas relacionadas con la práctica médica (incluidos los 11 egresados de la Real Universidad). 
Como vemos, Ylisaliturri no enseñó medicina. Cano se incorporó como maestro y siguió formando a los alumnos en el Hospital de Belén. Al parecer, Universidad e Instituto tenían ideas antagónicas; mientras aquella apoyaba a la Corona y sus estudiantes, quienes provenían de la elite, recibían lecciones dogmáticas, en el Instituto estaban inscritos alumnos de bienestar económico menor y poder precario que representaban la identididad regional, lo que en ese momento era igual a defender el régimen federalista (Cárdenas, 2007: 44). No obstante, observamos al Dr. Cano y Noreña como catedrático que estuvo presente en ambas instituciones, fue una pieza importante en ese periodo de transición. Las prácticas que tuvo con los alumnos nocturnos no desentonaron con el autodidactismo ni con la vida de tertulias, impulsados en el Instituto, y seguramente tuvo que adaptar su forma de enseñanza para moldear al nuevo ciudadano que reclamaba abrevar de los saberes ilustrados.

\section{Encargado de la conservación y propagación de la vacuna}

Enfermedades como el tifo, el cólera y la viruela asolaban con cierta frecuencia en distintas partes del mundo, y Guadalajara no fue la excepción. Nueva España recibió la vacuna en 1804, con financiamiento del rey de España Carlos IV y dirigida por el médico Francisco Xavier de Balmis. La vacunación se efectuaba "de brazo a brazo", es decir, se extraía la pus del brazo de un niño que ya había sido vacunado con anterioridad, el infante quedaba a cargo del facultativo encargado de dicha sustancia hasta que prendiera la pústula o grano vacunal; una vez madurado el grano, se extraía la pus para inocularlo de nuevo a otros niños, por ello era importante contar siempre con niños vacunados y lograr que las madres aceptaran la inoculación a toda costa. La viruela era endémica y se presentaba de manera cíclica (Agostoni, 2017: 23).

El procedimiento podía causar dolor y malestar y estaba rodeado de incertidumbre, dado que no se sabía de qué manera la vacuna operaba en el sistema inmunológico y encerraba múltiples peligros, siendo el contraer la sífilis vacunal uno de los más temidos (Agostoni, 2017: 24).

El Dr. Cano aprendió a aplicar y conservar la vacuna; incluso, al estar encomendado como facultativo del Hospital de Belén, le correspondió atender esta tarea a partir de 1819 hasta aproximadamente 1824. En este cargo enfrentó algunos problemas: la conservación y propagación del fluido vacuno era responsabilidad de los ayuntamientos o autoridades locales, y el presupuesto asignado para este rubro era muy reducido. El ayuntamiento de Guadalajara no fue la excepción. Por ello, en muchas ocasiones el Dr. Cano tuvo que sufragar algunos gastos de su propio pecunio, por ejemplo, la gratificación a las madres de los niños para que le permitieran 
vacunarlos ${ }^{35}$ o financiar los gastos para conseguir la sustancia de otros lugares cuando esta se perdía (Ruiz, 1991: 35; López, 1986: 71).

Así, por un oficio del propio Dr. Cano dirigido al ayuntamiento, tenemos noticia de cómo había recuperado con muchos sacrificios y a sus expensas la vacuna que se había perdido en el año de 1819. ${ }^{36}$ Posteriormente, a un año de haberla recobrado, informó a la Junta de Sanidad que esta estaba a punto de perderse nuevamente por la falta de niños en quienes inocular la pus, por lo que la Junta de Sanidad acordó avisar al ayuntamiento de la necesidad de promover el aumento y propagación de la vacunación para evitar su pérdida y hacían un llamamiento concreto:

[...] se pide al Ayuntamiento ordene que halla en el hospital al menos doce niños por el tiempo necesario para recoger el pus y se prevenga a los cuarteleros que cada uno presenten en el hospital un niño para escoger de los más sanos hasta completar el número adecuado[...]. ${ }^{37}$

En 1821, el Dr. José María Cano ocupó el cargo de regidor y miembro de la Junta de Sanidad en el ayuntamiento de la ciudad, por tal motivo, el 4 de enero de 1821se eligió al Dr. Pedro Figueroa como facultativo encargado de la conservación de la vacuna. ${ }^{38}$ La reacción de Noreña no se hizo esperar, pues en 1822 él argumentó que por su celo se había establecido la vacuna y que a sus expensas había traído el fluido pus y lo había mantenido bajo su cuidado. El conflicto se resolvió a su favor, el ayuntamiento corrigió su desatino al reconocer el agravio que esto significaba para Cano y lo restableció como encargado en un oficio fechado el 10 de julio de 1822, mientras que al Dr. Pedro Figueroa se le dieron las debidas gracias. ${ }^{39}$ Cano y Noreña se mantuvo en este cargo varios años más pues en un oficio del primero de marzo de 1824 aparece como médico titular del Hospital de Belén y encargado de la vacuna. ${ }^{40}$

Iniciando la tercera década, el 28 de enero de 1830 Cano y Noreña solicitó al Congreso del estado su jubilación a causa de una aneurosis, enfermedad que podría hacerle perder la vista (Horta, 2002, 86-87). Pero esto no representó un retiro total de sus actividades pues, durante el contagio masivo de viruela que azotó la ciudad este mismo año, se formó la Junta de Beneficencia para atender a la población. En el Acta de 14 de julio de 1830, sobre la instalación de la Junta

$35 \mathrm{Al}$ efectuarse la vacuna mediante la operación conocida "de brazo a brazo", que consistía en extraer la pus del brazo de un niño que ya había sido vacunado con anterioridad, este quedaba bajo el cuidado del facultativo encargado de dicha sustancia, hasta que prendiera la pústula o grano vacunal; una vez que estaba maduro este grano, se extraía la pus para inocularlo de nuevo a otros niños, por ello era importante contar siempre con niños vacunados y lograr que las madres aceptaran la inoculación a toda costa.

36 Documento sin título. Guadalajara, 1819. AGG. Paquete 39, leg. 229, f. 25.

37 Documento sin título. Guadalajara, 1820. AGG. Paquete 36, leg. 177, f. 3

38 Documento sin título. Guadalajara, 1821. AGG, paquete 36, Leg. 174, f. 4.

39 Documento sin título. Guadalajara, 1822. AGG, paquete 39, Leg. 229, fs. 24 y 24 vta.

40 Oficios relativos a la administración de la vacuna durante el año de 1824. Guadalajara, 1824. AGG, paquete 42, Leg. 250 , fs. 30. 
de Beneficencia para combatir dicha epidemia, se menciona al Dr. José María Cano como uno de sus miembros. ${ }^{41}$ Inmediatamente, el 16 de julio de 1830, la Junta acordó:

[...] que el Ayuntamiento nombre a los facultativos Dr. José María Cano, Pedro Figueroa y Luis Portugal para que acuerden y presenten un método curativo el más sencillo que puedan y les parezca el cual se imprima y circule en la capital y se extienda a todos los ayuntamientos del Estado. ${ }^{42}$

Igualmente, junto con el Dr. Nicolás Pérez, fueron comisionados como facultativos encargados de atender a los feligreses de la Parroquia del Sagrario en la curación de los enfermos (Ruiz, 1991: 74). Su participación fue muy activa en la Junta, incluso, por su experiencia como galeno, propuso que la junta no cerrara sino suspendiera momentáneamente sus sesiones pues estaba "persuadido" de que en la entrante estación se regeneraría la epidemia, y así se informó al ayuntamiento. ${ }^{43}$ Cano y Noreña fue uno de los partidarios de la instauración de la vacunación masiva, actividad que conforme avanzó la ciencia fue mejorando sus procedimientos y resultados.

\section{Clausura del Instituto y reapertura de la Universidad de Guadalajara}

Pocos años duró la vigencia del programa educativo pues la década de los treinta inició con cambios en materia educativa. El 1 de septiembre de 1834 fue clausurado el Instituto de Ciencias del Estado por el nuevo gobernador Antonio Romero y se restablecieron la Universidad y el Colegio de San Juan.

El 4 de septiembre de 1834, el Dr. José Cesáreo de la Rosa, quien fungía como rector cuando se clausuró la Universidad en 1826, convocó a los doctores universitarios que residían en la ciudad tras el decreto de su reapertura. Se nombró una diputación para que fuera con el gobernador del estado para agradecer el restablecimiento y dar testimonio de que se había reunido el claustro de doctores:

En seguida, y para el cumplimiento del artículo $3^{\circ}$ del expresado Superior Decreto, acordaron nombrar una comisión que forme el plan de estudios y proponga reformas en las constituciones de esta universidad, y la formaron por unanimidad de votos los Señores Dr. Pedro Espinoza, D. Ignacio García, D. Francisco Arroyo y D. José María Cano, asociando luego al Sr. D. Domingo Sánchez Reza [...]. ${ }^{44}$

41 Actas de la Junta de Beneficencia para la epidemia de viruela. Guadalajara, 1830. AGG. AY 3, paquete 51, Leg. 104, fs. 21. 42 Actas de la Junta de Beneficencia para la epidemia de viruela. Guadalajara, 1830. AGG. AY 3, paquete 51, Leg. 104, 21 fs. 43 lbíd.

44 Libro segundo del Claustro de Doctores. Guadalajara, 1834. AHUG. FRyLUG. Libro: 11, fs. 123v. 
La Universidad inició con las cátedras que se impartían anteriormente mientras se creaba un nuevo plan de estudios. Cano y Noreña, ya en calidad de catedrático decano, quedó como titular de la de Prima de Medicina; para la de Vísperas fue incorporado el cirujano latino y bachiller Ignacio Moreno, ${ }^{45}$ quien fungía como cirujano mayor del nosocomio de Belén, al lado de Cano. Dos años después fue retirado Ignacio Moreno por no haber obtenido los grados mayores en tiempo y forma, y en su lugar quedó José Antonio López.

En 1838 se creó la Sociedad Médica de Emulación de Guadalajara, organismo gremial que tuvo influencia en la trasformación de la práctica y la enseñanza médica, de la cual fue miembro (Horta, 2002: 83). En dicha sociedad participaron activamente los galenos que se habían formado en el Instituto de Ciencias y egresado de la antigua Universidad, que fueron alumnos de Cano: Antonio López, Francisco Garibay, Pablo Gutiérrez, encabezados por el gobernador recién depuesto, Pedro Tamez y Jurado, hijo del primer catedrático de cirugía de la antigua Universidad — también médico y alumno de Cano y Noreña-; el cirujano militar recién llegado a la ciudad, Pedro Vander Linden; el cirujano Ignacio Moreno, entre otros. Es para 1839, con la restructuración de la enseñanza médica que tenía la propuesta de unir la medicina con la cirugía, que nace la Escuela de Medicina, Cirugía y Farmacia. En esta restructura universitaria, el doctor Cano apoyó a algunos de sus exalumnos para ocupar las nuevas cátedras, como Pablo Gutiérrez y Antonio López, y no tuvieron cabida los egresados del anterior Instituto de Ciencias.

En este mismo periodo de reapertura de la Universidad, Cano fue nombrado consiliario para los bienios de $1835-1837,{ }^{46} 1837-1839,{ }^{47} 1843-1845,{ }^{48} \mathrm{y}$ finalmente para el de $1847-1849,{ }^{49}$ que tampoco concluye por sobrevenirle la muerte.

Además, ocupó el cargo de Diputado de Hacienda de la Universidad para el bienio de 1835$1837,{ }^{50}$ hay indicios de que también en el de 1837-1839 ya que se le menciona como miembro del claustro de hacienda en varias sesiones de estos años, y finalmente para el bienio de 18411843. Para este último periodo, al parecer fue muy reñida la elección por parte de los médicos, ya que además del Dr. José María Cano también se optaba por el Dr. Pablo Gutiérrez, pero definitivamente la decisión favoreció al Dr. Cano y Noreña. ${ }^{51}$

Otro de los cargos que tuvo en la Universidad fue el de Regente de la Escuela de Medicina, el cual cubrió en dos ocasiones; la primera fue nombrado el 14 de diciembre de 1843 ante la

45 Originario de la Barca, Jalisco. Se formó en el Real Colegio de Cirugía de la ciudad de México, al lado de Antonio Serrano. Después estudió medicina en la Real y Pontificia Universidad de México. Llegó a Guadalajara por el año de 1824, participó en la Comisión de Vacuna, ocupó cargos en el Ayuntamiento y fue Cirujano Mayor en el Real Hospital de San Miguel de Belén. Fue miembro de la Sociedad Médica de Emulación de Guadalajara. En: Méritos Literarios de Ignacio Moreno. Guadalajara, 1835. AHUG. FRyLUG. Caja 9, Exp. 179.

46 AHUG. Fondo Real y Literaria Universidad de Guadalajara. Libro Segundo de claustro de doctores, caja 33, Año 1825, fs. 137 vta. y 138.

47 Libro tercero de claustro de consiliarios. Guadalajara, 1837. AHUG. FRyLUG. Caja 29, fs. 6-9.

48 Libro tercero de claustro de consiliarios, Guadalajara, 1843. AHUG. FRyLUG. Caja 29, fs. 43 y 43 vta.

49 Libro tercero de claustro de consiliarios, Guadalajara, 1847. AHUG. FRyLUG. Caja 29, fs. 54 vta.

50 Libro Segundo de claustro de doctores, Guadalajara, 1835. AHUG. FRyLUG. Caja 33, fs. 150 vta.

51 Libro Tercero de claustro Mayores, Guadalajara, 1841.AHUG. FRyLUG. Caja 33, fs. 63.63 vta. 
renuncia del Dr. Luis Portugal;; 52 este cargo lo ocupó hasta el 11 de noviembre de 1845, fecha en que es nombrado el nuevo regente. Por segunda ocasión fue elegido el 11 de noviembre de 1847, pero no sabemos cuándo concluyó.

A sus 55 años de edad, con sus padecimientos de aneurosis, en el Claustro Universitario del 28 de noviembre de 1842 se leyó la solicitud de jubilación del Dr. José María Cano. ${ }^{53}$ Posiblemente ya para estas fechas le resultaba difícil acudir a sus labores pues en la sesión de consiliarios, fechada el 5 de diciembre de 1842, se trató el asunto de su jubilación, pero se suspendió la toma del acuerdo por encontrarse ausente el interesado. ${ }^{54}$ Aun así, Cano seguía realizando sus funciones de médico, pues en un oficio del 8 de enero de 1843, sobre el nombramiento de los profesores de medicina para los cuarteles de la ciudad, el Dr. José María Cano, junto con el Dr. Ildefonso Lazo, don Pablo Gutiérrez, don Nicolás Pérez y don Rafael Jiménez, fueron propuestos para ser encargados del cuartel № 1 por vivir en esa circunscripción. ${ }^{55}$

Dos años antes de su muerte fue retratado por el notable pintor José María Estrada, pintura que se conserva actualmente en el museo del estado (Porrúa, 1995: 581). Noreña murió en Guadalajara el 27 de septiembre de 1848 (Horta, 2002: 86-88).

\section{Consideraciones finales}

Para Cárdenas (2007: 50), la Real Universidad de Guadalajara surge como una reivindicación de la identidad de la Nueva Galicia frente al poder del virreinato centralizado en la ciudad de México. Eso hacía que los habitantes de la región se representaran a sí mismos como diferentes a los capitalinos. El modelo de enseñanza superior que encajaba con estas ideas era el universitario, aunque se tratara del avalado por la metrópoli. Después de la Independencia, cuando la Universidad se convirtió en Nacional, se diluyó esa identidad; luego, el Instituto de Ciencias introdujo algunos cambios en la enseñanza con libros novedosos, dejando de lado las cátedras basadas en "autoridades centenarias".

En este marco histórico nuestro personaje supo adaptarse. El doctor Carlos Ramírez (2004b: 7) nos dice de él que para el año de 1831 ejerció la cirugía con definiciones científicas y que realizaba con éxito amputaciones y desarticulaciones. A pesar de la novedad de la sistematización científica, debió haber sido una cirugía sin anestesia, los cirujanos en ropa de calle, con mala iluminación, en local inadecuado, y todo ello en medio del dolor del operado. Añade que el Dr. Cano fue una influencia definitiva para algunos facultativos célebres de generaciones posteriores, cita como ejemplos a los doctores Pablo Gutiérrez y Leonardo Oliva.

El mismo Dr. Oliva señala que las tareas que su maestro Cano y Noreña realizó fueron útiles a la ciencia que profesó; que con frecuencia les hacía notar lo mal montada que se veía la

52 Libro tercero de claustro de consiliarios, Guadalajara, 1843. AHUG. FRyLUG. Caja 29, fs.45-45 vta.

53 Libro tercero de claustro de Consiliarios, Guadalajara, 1842. AHUG. FryLUG. Caja 29, fs. 39-40.

54 Libro tercero de claustro de conciliarios, Guadalajara, 1842. AHUG. FRyLUG. Caja 29, fs. 40-41.

55 Documento sin título. Guadalajara, 1843. AGG. AY/ 11, caja 8, paquete 57, Leg. 77.

Diólo@os 
enseñanza médica y que tenía una noción clara de las reformas que deberían hacérsele, que contribuyó a las que se le hicieron, y añade:

Uno de nosotros, que practicó más de dos años bajo su dirección, tuvo oportunidad de apreciar por sí mismo su acierto en el tratamiento de las enfermedades, lo que justifica sobradamente la fama pública que gozó en el ejercicio civil de su profesión, mereciendo la confianza y preferencia de los primeros personajes del estado desde el tiempo del gobierno español, distinguiéndose entre sus apreciadores, el general D. José de la Cruz y el actual diocesano de este obispado (Ramírez, 2004b: 10).

En materia de educación le tocó vivir de cerca los siguientes cambios: el primer plan de estudios de la Universidad (1800-1816); el cambio de plan para adaptarlo a las Constituciones propias de la Universidad (1816-1826); el periodo del Instituto de Ciencias, donde además ejerció la docencia; la reapertura de la Universidad de Guadalajara, en la que inició como consiliario; la implantación del Plan Nájera; y la Reforma de 1839 e inmediatamente la reforma de 1840. Se retiró cuando se cerró nuevamente la Universidad y se reabrió el Instituto (1847-1848) que es cuando falleció. Y todo esto en diferentes marcos políticos: la época colonial, la independencia, el imperio de Iturbide y la construcción del modelo federal.

El Doctor José María Cano se encuentra entre los médicos que más cambios vivió; además, siendo partidario del realismo y de un fuerte sentido católico, pudo habérsele ubicado como conservador. Apoyó el desarrollo de la medicina en todos los ámbitos: en la enseñanza, en la práctica médica, en la conservación de la vacuna, participando activamente en Juntas de Sanidad, incluso llegó a presidir una de ellas. La mayoría de los médicos que impartieron sus cátedras durante el periodo de la Real Universidad fallecieron antes de su cierre; él sobrevivió y estuvo presente en el Instituto, sitio en el que se pretendía brindar una educación novedosa — no ya de dictar cátedras sino de explicar los contenidos de los libros-, y preparar a los estudiantes con libros modernos bajo los criterios de la ilustración.

Otro punto a considerar es que Cano fue un médico al que encontramos en el terreno médico, académico, político, lo que le generó numerosos vínculos de toda índole, tanto al interior de la Universidad, al ocupar puestos docentes y administrativos, así como en el Ayuntamiento de la ciudad, con sus cargos de regidor y miembro de la Junta de Sanidad, e incluso llegó a ser Diputado local en el Congreso del estado. Se vinculó también con la milicia al servir como cirujano en esa corporación en diversas ocasiones.

El papel que desempeñó en la conservación del fluido vacuno fue vital, sin su intervención se hubiera perdido esa sustancia; no le importó gastar de su bolsillo para su conservación, siempre estuvo muy interesado por la niñez. Tomó la conservación de la vacuna como una cuestión personal, no solo profesional, lo cual sin la menor duda habla bien de él, de sus valores éticos. 
La creación de la Academia nocturna para los estudiantes muestra otro rasgo de su carácter; se interesaba por allanar el camino de los jóvenes, suplir de alguna forma las carencias formativas de la Universidad en cuanto a clases, dotándolos de materiales que les sirvieran para ser mejores médicos; ponía a la disposición de ellos su biblioteca particular, se preocupaba por tener obras modernas de medicina, láminas para estudiar la anatomía.

El Dr. Oliva (Ramírez, 2004b: 10) señala que su sigilo profesional lo recomendaba pues nunca reveló el secreto de las consecuencias vergonzosas de las pasiones y deslices humanos; que jamás abandonó cruelmente a sus enfermos sino que le disputaba sus vidas a la muerte. Igualmente, nunca los dejó sin auxilios religiosos y fue sumamente caritativo.

La trayectoria laboral del Dr. José María Cano y Noreña es digna de ser conocida y difundida, e incluirla en los anales de la medicina del estado de Jalisco pues su obra ha quedado prácticamente en el olvido. Es justo que se rescaten sus aportaciones, independientemente de las ideas políticas que profesara.

\section{Referencias}

Agostoni, C. (2017). Médicos, campañas y vacunas de la viruela y la cultura de su prevención en México 1870-1952. Históricas Digital (Serie Historia Moderna y Contemporánea), (70), 2263. México: UNAM-Instituto de Investigaciones Históricas. http://www.historicas.unam.mx/ publicaciones/publicadigital/libros/medicos/660 0402 costumbres debates.pdf

Aguirre Salvador, R. (2003). El mérito y la estrategia: Clérigos, juristas y médicos en Nueva España. México: CESU-UNAM, Plaza y Valdez.

Anales de la Sociedad Médica de Emulación de Guadalajara (1838). Diciembre. Tomo I, (1-2).

Arias, P. (2000). El comercio. En: Martínez Réding, F. (dir.), Enciclopedia Temática de Jalisco. Tomo XIII. Guadalajara: Gobierno del Estado de Jalisco, 5-70.

Cárdenas Castillo, C. (1999). Aventuras y desventuras de la educación superior en Guadalajara durante el siglo XIX. Guadalajara: EDUG.

Cárdenas Castillo, C. (2007). Universidad, representaciones sociales e identidad regional en Guadalajara durante el siglo XIX. En Chavoya Peña, M. L. (coord.). Episodios de la Universidad de Guadalajara. Perspectivas diversas. Guadalajara: Universidad de Guadalajara, 41-52.

De la Torre De la Torre, F. (2006). Entre la quimera y la realidad: Cultura científico-tecnológica e industrialización en Jalisco en el siglo XIX. Tesis de Doctorado en Historia. Puebla: Benemérita Universidad Autónoma de Puebla-Instituto de Ciencias Sociales y Humanidades.

Gaceta Municipal de Guadalajara (1987). Gaceta Municipal de Guadalajara: (Documentos inéditos y monografías), s.n. Guadalajara: Municipio de Guadalajara.

Horta Rojas, J. (2002). La Sociedad Médica de Emulación de Guadalajara y la transformación de la enseñanza médica: 1837-1840. Tesis Maestría en Investigación en Ciencias de la Educación. Guadalajara: Universidad de Guadalajara. 
Iguíniz, J. B. (1992). Catálogo bibliográfico de los doctores, licenciados y maestros de la Antigua Universidad de Guadalajara. Guadalajara: EDUG.

López Almaraz, R. (1986). Epopeyas médicas de Guadalajara, en el siglo XIX. Guadalajara: UNED.

López Jiménez, J. (1988). Guadalajara y sus mandatarios. Guadalajara: Ayuntamiento de Guadalajara.

Martínez Moya, A. (2007). La educación universitaria en la intendencia de Guadalajara en la época de la Independencia de México. En Chavoya Peña, M. L. (coord.). Episodios de la Universidad de Guadalajara. Perspectivas diversas. Guadalajara: Universidad de Guadalajara, 17-39.

Muriá, J. (1982). Intendencia y provincia de Guadalajara. En Muriá, J. M.; J. Olveda; A. Dorantes; V. González (coords.). Lecturas históricas de Jalisco. Antes de la Independencia. Tomo I. Guadalajara: UNED, 363-382.

Parry, J. H. (1982). La administración de la Audiencia y cancillería real de Guadalajara (1572-1600). En Muriá, J. M.; J. Olveda; A. Dorantes; V. González (coords.). Lecturas históricas de Jalisco. Antes de la Independencia. Tomo I. Guadalajara: UNED, 267-290.

Peregrina, A. (1993). La educación superior en el Occidente de México. Tomo I, Siglo XIX, Guadalajara: Universidad de Guadalajara/El Colegio de Jalisco.

Porrúa (1995). Diccionario Porrúa de Historia, Biografía y Geografía de México. 6ta. Ed. México: Editorial Porrúa.

Porrúa (1995). Diccionario Porrúa de Historia, Biografía y Geografía de México. 6ta. Ed. Tomo A-C. México: Editorial Porrúa.

Ramírez Esparza, C. (2004a). Apuntes y recopilaciones bibliográficas para la historia del Hospital Civil de 1791 a 1950. Tomo I.

Ramírez Esparza, C. (2004b). Apuntes y recopilaciones bibliográficas para la historia del Hospital Civil de 1791 a 1950. Tomo II.

Riviere D'Arc, H. (1982). Las fases del crecimiento y del desarrollo de Guadalajara y de su región durante la colonización. En Muriá, J. M.; J. Olveda; A. Dorantes; V. González (coords.). Lecturas históricas de Jalisco. Antes de la Independencia. Tomo II. Guadalajara: UNED, 29-45.

Ruiz Briseño, G. (1991). La vacuna antivariolosa y la epidemia de viruela de 1830 en Guadalajara: Un estudio histórico Social. Tesis Licenciatura en Historia. Guadalajara: Universidad de Guadalajara.

Serrera Contreras, R. (1982). La industria pecuaria en Nueva Galicia a fines del siglo XVIII y principios del XIX. En Muriá, J. M.; J. Olveda; A. Dorantes; V. González (coords.). Lecturas históricas de Jalisco. Antes de la Independencia. Tomo II. Guadalajara: UNED, 105-110.

Viesca Treviño, C. (2010). Epidemias y enfermedades en tiempos de la Independencia. Rev Med Inst Mex Seguro Soc, 48(1): 47-54. https://www.medigraphic.com/pdfs/imss/im-2010/ im101i.pdf 
Viveros Ríos, O. (1999). Las prácticas y los saberes de los médicos en el siglo XIX (Guadalajara 18391888). Tesis Maestría en Investigación en Ciencias de la Educación. Guadalajara: Universidad de Guadalajara.

Villaseñor y Villaseñor, R. (1983). Bibliografía general de Jalisco. Tomo Il. Guadalajara: Unidad Editorial del Gobierno de Jalisco. 